informal credit-based arrangements - and were recruited using snowball and location-based methods in October-December 2010. We used successive logistic regression models to explore determinants of HIV infection among women reporting venue-based sex work (e.g. bars - includes women recruited onsite) and women reporting non-venue based sex work.

Results HIV prevalence was significantly higher in venue SW compared to non-venue women $(55.5 \%$ vs. $41.9 \%$, p < 0.01$)$. Numbers of clients reported were low, but more venue SW reported 2 or more clients in the last fortnight ( $38 \%$ vs. $7.6 \%, p<0.01)$; consistent condom-use during commercial acts was similar ( $60 \%$ and $68 \%, p>0.1)$. More venue SW reported ever travelling away from where they live to sell sex $(27 \%$ vs. $12 \%, \mathrm{p}<0.01)$, initiated sex work earlier (age 25 vs. 28 years, $p<0.01$ ), and sold sex for longer ( 6 vs. 5 years, $p<0.05$ ). Factors associated with HIV infection were type of sex work (aOR: 2.2, 95\% CI: 1.3-3.8 vs. non-venue), and SW not requesting condom-use (aOR: 3.0, 95\% CI: 1.15-7.85), after controlling for basic demographic differences.

Conclusion Venue SW were more likely to be HIV-positive than non-venue women, although in both groups HIV prevalence was substantial, and consistent condom use low. High risk of HIV among women not requesting condom use highlights the importance to renew prevention efforts in this population. The results emphasise the need to understand SW more broadly.

\section{P3.105 USING RESPONDENT-DRIVEN SAMPLING TO ESTIMATE HIV AND SYPHILIS PREVALENCE AMONG FEMALE SEX WORKERS IN AGADIR, FES, RABAT AND TANGIER, MOROCCO}

doi:10.1136/sextrans-2013-051184.0564

${ }^{1} \mathrm{~L}$ Johnston, ${ }^{2} \mathrm{~A}$ Bennani, ${ }^{2} \mathrm{~A}$ Latifi, ${ }^{3} \mathrm{H}$ Oumzil, ${ }^{4} \mathrm{~B}$ El Omari, ${ }^{5} \mathrm{~F}$ El Rhoufrani, ${ }^{6} \mathrm{~L}$ Ouarsas, ${ }^{7} \mathrm{~K}$ Alami, ${ }^{7} \mathrm{H}$ El rhilani. 'University of California, San Francisco, CA, United States; 'Ministry of health, NAP, Rabat, Morocco; ${ }^{3}$ Ministry of health, INH, Rabat, Morocco; ${ }^{4}$ Unité de gestion du Fonds Mondial, Rabat, Morocco; ${ }^{5}$ Association de Lutte Contre le Sida, Rabat, Morocco; ${ }^{6}$ Association de Lutte Contre le Sida, Agadir, Morocco; 7 UNAIDS, Rabat, Morocco

Background Throughout the world, including in the Middle East and North Africa region, female sex workers (FSW) often have a disproportionately high prevalence of HIV infection and they, along with their clients, are considered a core group contributing to the transmission of HIV in many countries. FSW, a highly stigmatised, hard-to-reach and understudied population in Morocco were surveyed in four cities in Morocco, using Respondent-Driven Sampling.

Methods 372 FSW in Agadir, 359 in Fes, 392 in Rabat and 324 in Tangier were sampled over the course of six to eight weeks in December 2011 and January 2012. Eligible females reported exchanging penetrative (vaginal/anal) sex for money with more than one male client in the past six months, being 18 years or older, holding Moroccan nationality and working in the respective study location. Estimates were calculated using the multiplicity estimator in RDSAT V.6.0.

Results Most FSW in all four cities had no or low education, were separated, divorced or widowed and financially supporting adults and/or children. HIV seroprevalence in Agadir was 5.1\%, in Fes $1.8 \%$ and in Tangier $1.4 \%$. No one tested positive for HIV in Rabat. Syphilis infection in Agadir was $21.4 \%$, in Fes $18.8 \%$, in Rabat $13.9 \%$ and in Tanger $13.3 \%$.

Conclusions HIV among FSW in Morocco was lower than expected and confirms a concentrated epidemic in Agadir. Findings of syphilis infection among FSW may indicate infection with other sexually transmitted infections (STI) that were not tested in this survey and remain undetected. A scale up of programmes to provide targeted HIV outreach and services to FSW is essential to control the further spread of HIV and other STI in this population and to clients and other sexual partners.

\section{P3.106 POSSIBLE LINKS IN HIV INFECTION BETWEEN FEMALE SEX WORKERS (FSW) AND PEOPLE WHO INJECT DRUGS (PWID): FINDINGS FROM A RESPONDENT DRIVEN SAMPLING SURVEY IN DAR-ES-SALAAM, TANZANIA}

doi:10.1136/sextrans-2013-051184.0565

${ }^{1} \mathbf{N}$ - Makyao, ${ }^{2} \mathrm{~A}$ Kangolle, ${ }^{2} \mathrm{G}$ Arthur, ${ }^{3} \mathrm{M}$ Kazaura, ${ }^{4} \mathrm{~S}$ Welty, ${ }^{1} \mathrm{~B}$ Kilama, ${ }^{2} \mathrm{M}$ Kibona, ${ }^{1} \mathrm{~A}$ Ramadhani, ${ }^{3}$ S Kamazima. 'National AIDS Control Programme, Dar es Salaam, Tanzania; ${ }^{2}$ Centers for Disease Control and Prevention, Dar es Salaam, Tanzania; ${ }^{3}$ Muhimbili University of Health and Allied Sciences, Dar es Salaam, Tanzania; ${ }^{4}$ University of California San Francisco, California, CA, United States

Background Findings from studies done by McCurdy et al. have indicated that HIV prevalence may be as high as $42 \%$ among PWID in Dar es Salaam. Studies of PWID have also suggested overlaps between FSW and PWID populations. We investigated a Dar es Salaam FSW population to better understand possible linkages between these populations.

Method We conducted a cross-sectional study of FSW in 2010 using respondent-driven sampling (RDS). Participants completed questionnaires on socio-demographic variables, sexual and other risk behaviours; and had HIV testing. We used descriptive weighted statistics and logistic regression analysis to explore associations with HIV.

Results Among 537 FSW enrolled, 518 were tested for HIV giving $31.4 \%$ (95\% CI: $25.6,38.5)$ prevalence overall. In bivariate analysis, HIV prevalence was higher among FSW who suspected that partners injected drugs, $46.9 \%$ (95\% CI: $31.5,64.1)$ as compared to FSW who did not suspected that partners injected drugs $23.2 \%$ (95\% CI: 16.5, 29.4).

FSW rarely reported using drugs themselves $1.3 \%$ (95\% CI: 0.3 , 2.7), but often suspected their partners were PWID (49.1\%; $95 \% \mathrm{CI}$ : $43.8,55.5)$. In a multivariate model, adjusting for demographic and behavioural characteristics, the adjusted odds ratio (AOR) of HIV infection among women who suspected that their partners injected drugs was 2.41 95\% CI: 1.22, 4.80; (1.4 times greater) and was 0.08 times higher per additional year of sex work (AOR 1.08 95\% CI: 1.03, 1.1)

Conclusion These findings provide supportive evidence of the connexion between FSW and PWID populations in Dar es Salaam. Considering these apparent connexions between PWID and FSW, we encourage programmes working with these two key populations to work together to achieve enhanced prevention outcomes.

\section{P3.107 RESULTS OF THE FIRST HIV PREVALENCE AND RISK BEHAVIOUR STUDY AMONG FEMALE SEX WORKERS, BELIZE, 2012}

doi:10.1136/sextrans-2013-051184.0566

'B Alvarez Rodríguez, ${ }^{2} \mathrm{M}$ Manzanero, 'S Morales Miranda. 'Universidad del Valle de Guatemala, Guatemala, Guatemala; ${ }^{2}$ Ministry of Health, Belmopan, Belize

Background In Central America, studies have been conducted in several countries to provide baseline estimates of the prevalence of human immunodeficiency virus (HIV) infection and sexually transmitted infections (STIs), and to elucidate behaviours associated with HIV/STIs among Female Sex Workers (FSW). This study reports the results from the first behavioural and biological surveillance survey conducted in Belize.

Methods We used a census-based approach to recruit FSWs in four districts of Belize. FSW at least 18 years of age who reported vaginal or anal sex in exchange for money during the previous 12 months were invited to participate in the study. Participants were administered a questionnaire through audio computer-assisted self-interview and tested for HIV, syphilis, herpes simplex virus type 2 (HSV-2), Treponema pallidum (TP), Chlamydia trachomatis (CT), Neisseria gonorrhoeae (NG), Trichomonas vaginalis (TV), and 
Mycoplasma genitalium (MG)). Proportions, medians, interquartile ranges (IOR), and 95\% confidence intervals were calculated using STATA 9.0.

Results A total of 220 FSWs were enrolled in the study. Median age was 25 years old (IOR: 21-30). Consistent condom use with clients in the last month was $81.33 \%$ (95\% CI: $74.16-87.22)$, and with occasional partners was $63.11 \%$ (95\% CI: $53.03-72.41)$. Approximately $57.27 \%$ (95\% CI: 50.45-63.9) had received an HIV test in the last 12 months. HIV prevalence was $0.91 \%(95 \%$ CI: $0.11-3.26 \%)$. The most prevalent STI was HSV-2 $(51.63 \%$, 95\% CI: 44.73-58.48), followed by CT; (19.79\%; 95\% CI: $14.33-$ $26.23)$, TV (18.18\%; 95\% CI: $12.93-24.47)$, and MG $(11.23 \%$; 95\% CI: 7.09-16.65). Syphilis and NG prevalence was observed at $<3 \%$.

Conclusions We found low prevalence of HIV in FSWs in Belize. However, risky sexual behaviours and STI prevalence remain a problem. Improved prevention strategies aimed at consistent condom use and access to HIV/STI testing are needed for the control and prevention of infections among FSW.

\section{P3.108 MOBILE GYNECOLOGICAL CLINIC - EFFECTIVE TOOL FOR HIVSTI SCREENING DIAGNOSTICS FOR COMMERCIAL SEX WORKERS IN LVIV REGION (UKRAINE)}

doi:10.1136/sextrans-2013-051184.0567

${ }^{1}$ M Vasylyev, ${ }^{1} M$ Sluzhynska, ${ }^{20}$ Sluzhynska, ${ }^{2} 0$ Grushynska, ${ }^{20}$ Krystynyak, ${ }^{2} 0$ Krystynyak, ${ }^{2} \mathrm{O}$ Krystynyak, ${ }^{2} \mathrm{O}$ Krystynyak, ${ }^{20}$ Krystynyak, ${ }^{20}$ Krystynyak. 'Lviv Regional AIDS Center, Lviv, Ukraine, ${ }^{2}$ Charitable Salus Foundation, Lviv, Ukraine

Background Since October 2008 till present time, Lviv Regional AIDS Center and Charitable Salus Foundation implement project "HIV prevention by increasing access to VCT (voluntary HIV testing and counselling), sexually transmitted infections (STI) diagnostics among commercial sex workers (CSW) in Lviv region using mobile gynaecological clinic (MGC). The initiative is supported by International HIV $\backslash$ AIDS Alliance in Ukraine.

Methods Specially equipped bus - MGC was used for this intervention. MGC works daily except Saturday and Sunday visiting highest HIV rate places all over Lviv region (2.5 million population) providing CSW with condoms, lubricants, information materials and rapid testing for HIV, syphilis, gonorrhoea, Chlamydia, hepatitis B and C. Shampoos, lipsticks, hand creams were distributed among CSW as a motivation for those clients who passed HIV \STI test.

Results During October 2008 - January 201312059 visits were done by CSW. 4031 rapid HIV test were made (197 positive results) 2886 rapid syphilis tests (37 positive results) 2133 tests for gonorrhoea (30 positive results) 2133 tests for Chlamydia (230 positive results) 1499 Hepatitis B tests (63 positive results) 1499 Hepatitis C tests (141 positive results)

Conclusions Taking into consideration obtained data such intervention is very needed and welcomed by key population representatives. Gender oriented approach in HIV prevention programmes for female sex workers is very important factor for the success of the intervention In great importance continuation of performed services and spreading the range of activities such as tuberculosis diagnostics, access to medications for treatment in MGC.

\section{P3.109 VARIABILITY IN THE DETERMINANTS AND PREVALENCE OF HIV AND SYPHILIS AMONG FEMALE SEX WORKERS IN TWO NEIGHBOURING DISTRICTS IN NORTH KARNATAKA INDIA}

doi:10.1136/sextrans-2013-051184.0568

${ }^{1}$ R G Washington, ${ }^{2} \mathrm{~N}$ Chandiok, 'P Banandur, ${ }^{1} \mathrm{~A}$ Krishnamurthy, ${ }^{1} \mathrm{G}$ Pise. ${ }^{1} \mathrm{KHPT}$, Bangalore, India; ${ }^{2}$ CMR, New Delhi, India
Background Districts in north Karnataka have the highest HIV prevalence and female sex worker (FSW) to male population ratio in India. We examined the variability in socio-cultural, behavioural and clinical determinants contributing to HIV and syphilis prevalence in two neighbouring north Karnataka districts.

Methods FSW recruited from targeted intervention clinics responded to an oral questionnaire, underwent clinical examination and provided a blood sample for HIV and syphilis testing. We conducted univariate and multivariate logistic regression with HIV and syphilis as outcomes and with age, place of residence, marital status, literacy, monthly income, alcohol use, age at sexual debut and commercial sex work, clientele over the past week and STI syndromes as variables.

Results 1545 and 1551 FSW were recruited in the two districts. HIV ( $8 \%$ vs $6 \%$ ) and syphilis prevalence ( $3 \%$ vs $1 \%$ ) was significantly higher in district A. District A had significantly higher proportions of rural residents ( $92 \%$ vs $81 \%$ ), devadasi ( $67 \%$ vs $15 \%$ ), illiteracy ( $80 \%$ vs $64 \%$ ), higher income (Rs6000 vs Rs 5000 ), clientele per week (6 vs 4 ) and STI syndromes (16\% vs $7 \%$ ). While age at sexual debut was similar in both districts, significantly higher proportion in district $A$ initiated sex work below the age of $15(26 \%$ vs $11 \%)$. Alcohol consumption was higher in B (15\% vs $3 \%)$. The odds of being HIV positive was significantly higher amongst FSWs with income > Rs5000 per month (AOR 1.61), being devadasi (AOR $2.43)$, consuming alcohol (AOR 1.64), h/o STI syndrome in the past 6 months (AOR 1.97) or clinically with an STI (1.95).

Conclusion Despite being neighbouring districts with similar socio-demographic backgrounds, significant variability in the prevalence of HIV and syphilis is perhaps contributed by the variability in prevalence of specific socio-cultural factors, behaviours and clinical syndromes of STI.

\section{P3.110 RELATIONSHIP BETWEEN VIOLENCE AND HIV INFECTION AMONG FEMALE SEX WORKERS IN BENIN}

doi:10.1136/sextrans-2013-051184.0569

1,2F K Tounkara, ${ }^{3} \mathrm{~F}$ Guédou, ${ }^{4} \mathrm{C}$ Ahoussinou, ${ }^{5} \mathrm{D}$ M Zannou, ${ }^{6} \mathrm{~A}$ Kpatchavi, ${ }^{7} \mathrm{D}$ D Kintin, ${ }^{8} \mathrm{~L}$ Avery, ${ }^{9} \mathrm{E}$ Bédard, ${ }^{1,2} \mathrm{R}$ Bitera, 1,2M Alary. 'Département de médecine sociale et préventive, Université Laval, Quebec, OC, Canada; ${ }^{2} U R E S P$, Centre de recherche du CHU, Quebec, OC, Canada; 'ispensaire des Infections Sexuellement Transmissibles (DIST), Cotonou, Benin; ${ }^{4}$ Programme nationale de lutte contre le Sida et les IST, Cotonou, Benin; ${ }^{5} 5$ Faculté des Sciences de la Santé, Université d'Abomey-Calavi, Cotonou, Benin; ${ }^{6}$ Département de sociologie, Université d'Abomey-Calavi, Cotonou, Benin; 'Bureau d'appui en santé publique (BASP'96), Cotonou, Benin; ${ }^{8}$ Faculty of

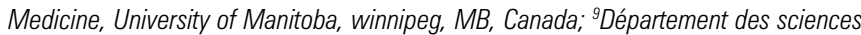
infirmières, Université du Québec à Rimouski, Campus Lévis, Lévis, OC, Canada

Objective In Africa, there are few studies examining female sex workers (FSWs) vulnerability to physical, sexual and psychological violence and how this influences their HIV risk. This study sought to assess the types, frequency and factors associated with violence among FSWs and examined the relationship between violence and HIV prevalence.

Methods Data from an integrated biological and behavioural survey (Benin, 2012), were used to analyse the frequency of physical, sexual and psychological violence. We also created a violence score using the number of violence types experienced by FSWs. Multivariable logistic regression analysis controlling for potential socio-demographic and behavioural confounders was used to assess the association between violence and HIV.

Results Among the 1016 FSWs recruited in this survey, 17.1\%, $13.3 \%$ and $33.4 \%$ reported having experienced, at least once in the past 30 days, physical, sexual and psychological violence, respectively. Condom breakage was strongly and significantly associated with all 3 types of violence $(P<0.0001)$. HIV prevalence was $20.39 \%$. In the multivariable analysis, the adjusted odds ratio (AOR) for the 